\title{
Application of BP Neural Network Model in Sports Aerobics Performance Evaluation
}

\author{
Shuhe Shao
}

\begin{abstract}
This article provides partial evaluation index and its standard of sports aerobics, including the following 12 indexes: health vitality, coordination, flexibility, accuracy, pace, endurance, elasticity, self-confidence, form, control, uniformity and musicality. The three-layer BP artificial neural network model including input layer, hidden layer and output layer is established. The result shows that the model can well reflect the non-linear relationship between the performance of 12 indexes and the overall performance. The predicted value of each sample is very close to the true value, with a relative error fluctuating around of 5\%, and the network training is successful. It shows that BP network has high prediction accuracy and good generalization capacity if being applied in sports aerobics performance evaluation after effective training.
\end{abstract}

Keywords-BP neural network, sports aerobics, performance, evaluation.

\section{INTRODUCTION}

$\mathrm{H}$ OW to evaluate the performance of sports aerobics is an issue worth studying, and many scholars have studied the evaluation method for course performance[1]. The usual method is to make scoring rule, in which the judge marks according to the completion of the rule, and adds up the scores. However, this method is greatly affected by the experience and level of the judge. AHP method is widely used in various types of performance evaluation. In comparison with traditional direct scoring method, this method achieves significant progress, combining the qualitative evaluation with the quantitative evaluation, and improves the accuracy of the evaluation.

BP (Back Propagation) network was proposed by the scientist team headed by Rumelhart and McCelland in 1986, which is a multi-layer feed-forward network trained by error back-propagation algorithm. BP network is currently one of the most widely applied neural network models [2]. BP network can learn and store a large number of inputs - output model mapping without disclosing or describing the mathematical equation of this mapping in advance. This method has wide application prospect in the performance evaluation of sports aerobics [3].

\section{METHOD}

Wherever the performance evaluation of sports aerobics can be divided into single event performance and overall performance. It is usually believed that the single event performance is more rational while the overall performance is more emotional, and the single event performance focuses on the details while the overall performance focus on the overall performance. However, in the actual operation, the single event

Shuhe Shao is with the Henan Institute of Science and Technology, Xinxiang, China P.R. (phone: 13849362681; fax: 0373-3040786; e-mail: qianguoximi@163.com). performance may be high while the overall performance is low, or the single event performance is at moderate level while the overall performance is very high. At this moment, BP neural network model can be used to identify the implication relation between the single event performance and overall performance, forming neural network model. After a lot of trainings, the expert only marks single performance in the performance evaluation, and the model automatically calculates the overall performance after error correction, which can improve the accuracy of evaluation.

The undermost layer in the three-layer BP artificial neural network model is known as input layer, the middle layer is hidden layer, and the upmost layer is output layer. The nerve cells among the layers are interconnected, and the nerve cells within the layers are not interconnected. The learning process of BP algorithm is composed of forward propagation and backward propagation. In the forward propagation, the information is propagated from input layer to output layer through hidden layer; and if the expected output result cannot be obtained in the input, the information will be transferred into backward propagation, returning the error signal along the original connecting path layer. The weight value adjustment of the model adopts the learning algorithm of backward propagation. The specific algorithm steps are as below [4]:

\section{Step 1 Input layer data}

Create sample set and factor set $\mathrm{x}_{\mathrm{i}}$.

\section{Step 2 Standardization of original data}

The node value in the neural network is defined between $0-1$, if some node value in the input layer is 0 , the information input from this node will not be propagated to the hidden layer and the output layer. To avoid this, the input value should be standardized, and be calculated as per formula (1).

Step 3 Confirmation of node number in the hidden layer

The initial value of node number in the hidden layer can be confirmed as per one of the following two formulas:

$$
1=\sqrt{0.43 m n+0.12 n^{2}+2.54 m+0.77 n+0.35+0.51}
$$

where: $\mathrm{m}, \mathrm{n}$ are the number of input nodes and number of output nodes; $a$ is the constant in between 1-10. An initial value of node number in the hidden layer can be obtained as per formula (1) or (2), and the stepwise increase method (starting with a simple network, and gradually increasing the cell number in the hidden layer till being suitable) or stepwise pruning method (starting with a complex network and gradually deleting the cell in the hidden layer) are utilized to solve. 
Step 4 Confirmation of learning error amount

The network output error is set as:

$$
E_{k}=\frac{1}{2} \sum_{j}\left(y_{j k}-o_{j k}\right)^{2}
$$

where: $y_{j k}$ and $O_{j k}$ are respectively the expected output and actual output of nerve cell $\mathrm{j}$. $\mathrm{E}=\Sigma \mathrm{Ek}$ is configured as the sum of output error produced by all samples in the whole training.

\section{Step 5 Transfer function}

In some circumstances, the pureline function can be adopted. If the final layer of BP network is Sigmoid function, the output of the entire network will be limited within a relatively small range (a continuous scale between 0 to 1 ); if the final layer of BP network is Pureline function, the output of the entire network can take any value.

\section{Step 6 Weight value adjustments}

The formula for weight value adjustment is:

$$
\Delta_{k} w_{j i}=\eta \delta_{k} O_{i k} \quad \eta>0
$$

where: $\Delta_{k} w_{j i}$ is the adjusted value of $\mathrm{w}_{\mathrm{ji}} ; \eta$ is learning rate, or known as step size.

If $\mathrm{j}$ is the output nerve cell of neural network:

$$
\delta_{j k}=\left(y_{j k}-o_{j k}\right) f^{\prime}\left(n e t_{j k}\right)
$$

If $\mathrm{j}$ is not the output nerve cell of neural network:

$$
\delta_{j k}=f^{\prime}\left(\text { net }_{j k}\right) \sum \delta_{m k} w_{m j}
$$

In the formula(6), net ${ }_{j k}=\sum_{i} w_{j k} o_{i k}$

\section{APPLICATION}

\section{A. Index selection}

According to the sports characteristics of sports aerobics, the main single event performance standard is divided into the following 12 indexes: health vitality, coordination, flexibility, accuracy, pace, endurance, elasticity, self-confidence, form, control, uniformity and musicality. See the specific evaluation standard in Table I.

\section{B. Confirmation of Sample}

30 students of aerobics major in sports department were selected as samples. The evaluation of single event score and total score are made by the teacher engaged in aerobics

\begin{tabular}{|c|c|}
\hline Index & Standard \\
\hline Health vitality & $\begin{array}{l}\text { The whole set of movements show the active, natural, and } \\
\text { dynamic, mental outlook }\end{array}$ \\
\hline Coordination & $\begin{array}{l}\text { The capacity of body and limbs of a player on completing } \\
\text { movement synchronously }\end{array}$ \\
\hline Flexibility & Capacity of moving amplitude of a player \\
\hline Accuracy & $\begin{array}{l}\text { Accuracy of the movement pattern position shown by } \\
\text { limbs }\end{array}$ \\
\hline Pace & Quality to complete five types of paces \\
\hline Endurance & $\begin{array}{l}\text { Capacity to keep exercise intensity of cardiovascular } \\
\text { system in the whole set of movements (non-intermittent) }\end{array}$ \\
\hline Elasticity & $\begin{array}{l}\text { Springiness and legerity shown by the player in the process } \\
\text { of completing movements }\end{array}$ \\
\hline Self-confidence & $\begin{array}{l}\text { Capacity to believe in himself/herself shown by the player } \\
\text { in the process of completing movements }\end{array}$ \\
\hline Form & $\begin{array}{l}\text { The whole set of movements must show correct movement } \\
\text { and standard body position }\end{array}$ \\
\hline Control & $\begin{array}{l}\text { Capacity of the player to control and regulate body in the } \\
\text { process of completing some movement }\end{array}$ \\
\hline Uniformity & $\begin{array}{l}\text { Uniformity of all players in completing movements at the } \\
\text { same time }\end{array}$ \\
\hline Musicality & Perfect combination of movement of the player with music \\
\hline
\end{tabular}
teaching, taking the mean value. See Table II.
TABLE I

Single EVent Performance Evaluation Index of Sports Aerobics

\section{Model Creation}

The network model adopts the three-layer BP network, and the input layer includes 12 nodes. According to formula 3, through calculation and adjustment, 12 nerve cells are selected in the hidden layer, input layer has one node $y$; the transfer function takes sigmoid; the maximum training parameter is set as 9000 ; the network training objective is error $<0.001$; the learning efficiency is 0.16 ; the momentum factor is 0.7 ; other parameters adopt default value. Calculate according to step 1-6, see the result in Table III.

\section{RESUlt AND ANALYSIS}

1) The network training will be successful if the predicted value of each sample is very close to the true value.

2) BP network will have high prediction accuracy and good generalization capacity after being applied in performance evaluation of sports aerobics through effective training if the relative error fluctuation is around $5 \%$.

3) The sample amount of network training should be increased to improve the accuracy of network training.

\section{CONCLUSION}

1) There is complex non-linear relation between health vitality, coordination, flexibility, accuracy, pace, endurance, elasticity, self-confidence, form, control, uniformity and musicality of sports aerobics and overall performance, and it is feasible to use BP neural network method for prediction.

2) The algorithm of network training model should be selected according to the actual situation, which should guarantee the stability of network and consider the convergence rate and learning time.

3) Somatic neural network model does not only have good application value in sports aerobics evaluation, but also has wide application prospect in performance evaluation of other sports events. 
TABLE II

MARKING RESULTS OF SPORTS AEROBICS EXPERTS

\begin{tabular}{|c|c|c|c|c|c|c|c|c|c|c|c|c|c|}
\hline & $\mathrm{X}_{1}$ & $\mathbf{X}_{2}$ & $\mathbf{X}_{3}$ & $\mathrm{X}_{4}$ & $X_{5}$ & $X_{6}$ & $X_{7}$ & $\mathrm{X}_{8}$ & $\mathrm{X}_{9}$ & $X_{10}$ & $X_{11}$ & $X_{12}$ & $\mathbf{Y}$ \\
\hline Sample & $\begin{array}{l}\text { Health } \\
\text { vitality }\end{array}$ & Coordination & Flexibility & Accuracy & Pace & Endurance & Elasticity & $\begin{array}{c}\text { Self- } \\
\text { confidence }\end{array}$ & Form & Control & Uniformity & Musicality & $\begin{array}{l}\text { Total } \\
\text { score }\end{array}$ \\
\hline 1 & 8.5 & 8.2 & 8.9 & 9 & 8.2 & 8.5 & 8.2 & 9.1 & 9.2 & 8.2 & 9.2 & 9.8 & 8.9 \\
\hline 2 & 9 & 9 & 8.2 & 9.8 & 9.3 & 8.5 & 9.5 & 9.8 & 9.8 & 8.2 & 8.2 & 8.8 & 9.5 \\
\hline 3 & 9.2 & 8.8 & 8.8 & 9.3 & 9.5 & 8.6 & 8.5 & 8.5 & 9.4 & 8.8 & 8.5 & 9.2 & 9 \\
\hline 4 & 8.2 & 8.4 & 9 & 8.2 & 8.6 & 8.5 & 8.5 & 9.3 & 8.2 & 8.5 & 9.1 & 9.3 & 8.6 \\
\hline 5 & 7.8 & 7.9 & 6.8 & 7.9 & 7.7 & 6.8 & 6.9 & 8.1 & 7.6 & 7.8 & 7.8 & 8.2 & 7.8 \\
\hline 6 & 8.6 & 9 & 9 & 8.8 & 8.5 & 7.9 & 8.6 & 8.5 & 8.2 & 8.5 & 9 & 8.8 & 8.6 \\
\hline 7 & 8.2 & 8.6 & 8.5 & 8.5 & 8.3 & 8.2 & 8.5 & 9.1 & 9.3 & 8.5 & 8.2 & 8.6 & 8.5 \\
\hline 8 & 8.9 & 8.7 & 8.8 & 8.9 & 9.1 & 9 & 8.8 & 8.8 & 9.2 & 8.9 & 9 & 9.5 & 8.9 \\
\hline 9 & 9.8 & 9.5 & 9.9 & 9.6 & 9.5 & 8.9 & 9.5 & 9.6 & 9.8 & 9.2 & 9.8 & 9.8 & 9.6 \\
\hline 10 & 9.8 & 9.3 & 8.5 & 8.9 & 8.9 & 8.5 & 8.2 & 9.2 & 9.4 & 9.5 & 9.1 & 9 & 9.5 \\
\hline 11 & 8.3 & 8.5 & 8.6 & 8.5 & 9 & 9 & 9 & 8.7 & 8.6 & 8.5 & 8.9 & 8.6 & 8.7 \\
\hline 12 & 8.2 & 8.6 & 8.5 & 8.5 & 8.5 & 9.2 & 8.8 & 8.8 & 9 & 8.6 & 9 & 9 & 8.8 \\
\hline 13 & 8.9 & 8.7 & 8.8 & 8.6 & 8.5 & 8.2 & 8.4 & 9 & 9 & 8.7 & 8.9 & 8.8 & 8.9 \\
\hline 14 & 9.8 & 9.5 & 9.8 & 9.6 & 9.5 & 9.5 & 9.5 & 9.8 & 9.8 & 9.3 & 9.6 & 9.8 & 9.7 \\
\hline 15 & 7.8 & 7.9 & 7.9 & 7.6 & 7.8 & 7.2 & 7.9 & 7.8 & 7.3 & 7.6 & 7.8 & 7.9 & 7.8 \\
\hline 16 & 9.3 & 9.8 & 9.8 & 9.2 & 9.6 & 9.5 & 9.4 & 9.5 & 9.6 & 9.5 & 9.5 & 9.5 & 9.5 \\
\hline 17 & 8.5 & 8.5 & 8.6 & 8.8 & 8.5 & 8.6 & 8.8 & 8.2 & 8.9 & 8.7 & 8.8 & 8.9 & 8.7 \\
\hline 18 & 8.6 & 8.3 & 8.2 & 8.5 & 8.5 & 8.2 & 8.9 & 8.8 & 8.8 & 8.5 & 8.9 & 8.6 & 8.6 \\
\hline 19 & 6.7 & 6.8 & 6.9 & 6.8 & 6.5 & 6.9 & 6.8 & 6.5 & 6.8 & 6.8 & 6.9 & 6.9 & 6.9 \\
\hline 20 & 9.5 & 9.5 & 8.9 & 9.5 & 9.2 & 9.5 & 9.6 & 9.2 & 9.5 & 9.2 & 9.5 & 9.3 & 9.5 \\
\hline 21 & 9.3 & 8.9 & 8.9 & 8.9 & 9.2 & 9.4 & 9.2 & 9 & 9.3 & 9 & 9.2 & 9.2 & 9.2 \\
\hline 22 & 8.1 & 8.5 & 8.2 & 8.5 & 8 & 8.9 & 8.8 & 8.8 & 8.6 & 8.8 & 8.8 & 8.5 & 8.7 \\
\hline 23 & 9.5 & 9.6 & 9.3 & 9.2 & 9.6 & 9 & 9 & 9.4 & 9.4 & 9.4 & 9 & 9.8 & 9.5 \\
\hline 24 & 9.1 & 9.2 & 8.8 & 8.8 & 9 & 8.8 & 9.5 & 8.9 & 8.9 & 8.9 & 9.1 & 9 & 9.1 \\
\hline 25 & 8.8 & 8.2 & 8.4 & 9 & 9 & 8.2 & 8.5 & 8.8 & 8.6 & 9 & 9 & 8.5 & 8.9 \\
\hline 26 & 8.6 & 8.5 & 8.9 & 8.8 & 8.9 & 9 & 8.5 & 8.5 & 9.1 & 8.8 & 8.6 & 8.5 & 8.7 \\
\hline 27 & 6.9 & 6.8 & 6.9 & 6.9 & 6.8 & 7.1 & 7 & 7 & 7.5 & 6.8 & 6.8 & 6.7 & 7.1 \\
\hline 28 & 8.2 & 8.2 & 8.5 & 9.1 & 8.2 & 8.6 & 8.5 & 8.5 & 8.5 & 8.6 & 8.8 & 8.8 & 8.7 \\
\hline 29 & 8.8 & 9 & 8.8 & 8.8 & 8.9 & 8.7 & 8.8 & 8.9 & 8.6 & 8.2 & 8.5 & 8.9 & 8.9 \\
\hline 30 & 8.8 & 8.9 & 8.6 & 8.9 & 8.9 & 8.5 & 8.9 & 8.6 & 8.1 & 9 & 8.8 & 9 & 8.9 \\
\hline
\end{tabular}


World Academy of Science, Engineering and Technology International Journal of Medical and Health Sciences Vol:6, No:12, 2012

TABLE III

Evaluation of True VAlue and PREdicted VAlue

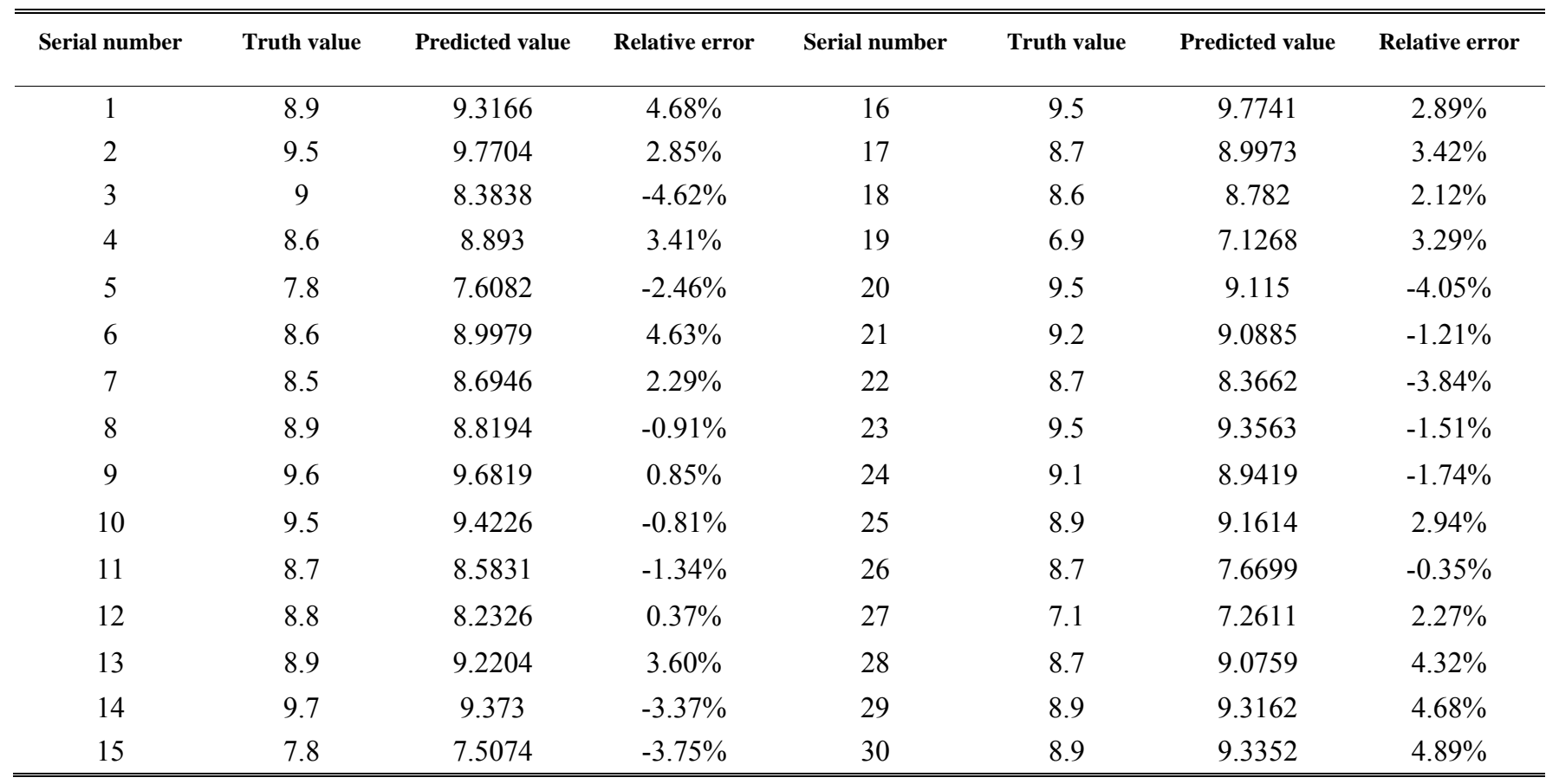

\section{REFERENCES}

[1] Meng Yueting and Wu Lili.Review and Prospects of Public Aerobics in China. Journal of Hubei Sports Science.2008(1):64.(in chinese)

[2] Du Fuzhi,Fu Wa-li and Du Xiao-hong.Water Quality Evaluation of Smal Basin in Three Gorges Reservoir Based on BP Neural Networks. Water Saving Irrigation .2009(1):8. (in chinese)

[3] Wang Yang,Yu Dinghua and Sun Peng.Simulation of preparation of acrylic acid from lacticacid dehydration based on BP neural network. CIESC Journal. 2009(1):83. (in chinese)

[1] Zhou Guo-liang,Liu Xi-yu and Wu Lu-ying. Application of BP neural network model on air quality rank appraisal. Computer Engine ring and Design. 2009,30 (2):392. (in chinese) 